Coupling discharge and gas dynamics in streamer-less spark formation in supercritical $\mathrm{N}_{2}$

This content has been downloaded from IOPscience. Please scroll down to see the full text.

2016 Jpn. J. Appl. Phys. 55 07LD06

(http://iopscience.iop.org/1347-4065/55/7S2/07LD06)

View the table of contents for this issue, or go to the journal homepage for more

Download details:

IP Address: 192.16.191.140

This content was downloaded on 30/03/2017 at 13:15

Please note that terms and conditions apply.

You may also be interested in:

Simulation of the hydrodynamic expansion following a nanosecond pulsed spark discharge in air at atmospheric pressure

Fabien Tholin and Anne Bourdon

Self-consistent modelling of charged and neutral particle dynamics in short-gap helium and hydrogen discharges

M Jugroot, $P$ Bayle, M Yousfi et al.

Modelling of a nanosecond surface discharge actuator

T Unfer and $\mathrm{J} P$ Boeuf

Neutral heating in glow to spark transition in air and nitrogen

$P$ Bayle, M Bayle and G Forn

Multi-scale modelling of pulsed nanosecond dielectric barrier plasma discharges in plane-to-plane $\underline{\text { geometry }}$

Sharath Nagaraja, Vigor Yang and Igor Adamovich

Nanosecond repetitively pulsed discharges in N2-O2 mixtures: inception cloud and streamer emergence

She Chen, L C J Heijmans, Rong Zeng et al.

Diffusion correction to the Raether-Meek criterion

Carolynne Montijn and Ute Ebert

Plasma predictions: past, present and future

J J Lowke 


\title{
Coupling discharge and gas dynamics in streamer-less spark formation in supercritical $\mathbf{N}_{2}$
}

\author{
Ashutosh Agnihotri ${ }^{1 *}$, Willem Hundsdorfer ${ }^{1,2}$, and Ute Ebert ${ }^{1,3}$ \\ ${ }^{1}$ Centrum Wiskunde \& Informatica (CWI), P. O. Box 94079, 1090 GB Amsterdam, The Netherlands \\ ${ }^{2}$ Institute for Mathematics, Astrophysics and Particle Physics (IMAPP), Faculty of Science, HEF 79, Radboud University, \\ P. O. Box 9010, 6500 GL Nijmegen, The Netherlands \\ ${ }^{3}$ Department of Applied Physics, Eindhoven University of Technology, P. O. Box 513, 5600 MB Eindhoven, The Netherlands \\ *E-mail: A.Agnihotri@cwi.nl
}

Received December 4, 2015; revised March 9, 2016; accepted March 26, 2016; published online June 15,2016

A two-dimensional cylindrically symmetric model is developed to study the streamer-less spark formation in a short gap on the timescale of ion motion. It incorporates the coupling between the electric discharge and the gas through the heat generated by the discharge and the consecutive gas expansion. The model is employed to study electrical breakdown in supercritical $\mathrm{N}_{2}$. We present the simulation results of gas heating by the electrical discharge and the effect of gas expansion on the electrical discharge. (C) 2016 The Japan Society of Applied Physics

\section{Introduction}

Electrical breakdown and dielectric recovery are important issues in high-voltage switch gear. A common medium in gaseous insulation and high-voltage switching is $\mathrm{SF}_{6}$ which is known to have excellent switching properties. ${ }^{1-4)}$ But the downside is that $\mathrm{SF}_{6}$ is an extreme green house gas with a global warming potential of 23900 times that of $\mathrm{CO}_{2}$. Supercritical liquids might be a replacement due to their high density, dielectric strength etc. ${ }^{5,6)}$ The electric breakdown in supercritical $\mathrm{N}_{2}$ - at 80 bar and $300 \mathrm{~K}$ - has recenty been studied experimentally and theoretically by Zhang et al. ${ }^{7)}$ However, in their simulation studies of the breakdown of supercritical nitrogen between two planar electrodes only the transversal structure of the breakdown channel was resolved in an extremely simplified model for the electric discharge. We here study the breakdown process assuming that the breakdown channel is cylindrically symmetric, i.e., that we can treat the evolution in a radial and a longitudinal coordinate $(r, z)$.

Electric breakdown is typically assumed to evolve from initial electron avalanches through the space charge dominated streamer phase to a heat dominated leader or arc phase, and many experimental and theoretical studies have been performed. ${ }^{8,9)}$ Space charge effects refer to the electric field enhancement at streamer head which supports the ionization reaction and thereby results in the subsequent breakdown of the medium. ${ }^{10,16)}$ Here we demonstrate a different breakdown mechanism in short gaps with secondary emission from the cathode where a sequence of ionization waves and ion pulses heats the gas up until it breaks down.

To study this dynamics, we here develop a simulational code to study electrical breakdown where discharge and gas dynamics are coupled: the discharge generates ohmic heat that heats the gas. Consecutively the gas expands which changes the transport and reaction properties in the discharge.

Tholin et al. ${ }^{11)}$ studied the hydrodynamic expansion of the background gas after the voltage pulse. They assumed the reduced electric field $E / N$ to be not affected by the changes in the background gas density, $N$, and hence do not solve the gas flow equations along with the discharge equations. This assumption is reasonable as the gas temperature does not rise rapidly during the short voltage pulse and hence the gas density is not much changed.

Komuro et al. ${ }^{12)}$ solve the discharge equations and background gas flow equations simultaneously during the voltage pulse, but only on the electron timescale.

In this work, we solve the discharge equations and background gas flow equations simultaneously but now on the timescale of ion motion.

This paper is organized as follows: In Sect. 2 we introduce the notation and describe our model for the coupled system. Section 3 contains initial and boundary conditions and numerical algorithms. Section 4 is devoted to simulation results and discussion. Finally, we summarize our finding and give an outlook in Sect. 5 .

\section{Description of the model}

We develop a two-dimensional (2D) cylindrically symmetric code to carry out the simulations. The code components include a model for the electric discharge on the timescale of ion motion and a model for the background gas. The two models are coupled to study the interplay between the electric discharge and the background gas.

\subsection{Model for the electric discharge}

To describe the discharge dynamics, we adopt the first-order reaction-drift-diffusion model ${ }^{13)}$ in local field approximation for electrons and ions, coupled to Poisson's equation to calculate the electric field self-consistently. We focus on the timescale of ion motion and assume that the electrons are moving infinitely fast. ${ }^{14,15)}$ This means that the electron density adapts to the ion density infinitely fast and the derivative $\partial_{\mathrm{t}} n_{\mathrm{e}}$ can be set to zero in an adiabatic decoupling procedure. The range of validity of this approximation will be discussed in a forthcoming paper. Hence, our reaction-driftdiffusion equations become:

$$
\begin{aligned}
& -\nabla \cdot\left(n_{\mathrm{e}} \mu_{\mathrm{e}} \mathbf{E}+D_{\mathrm{e}} \nabla n_{\mathrm{e}}\right)=S_{\mathrm{e}}, \\
& \partial_{\mathrm{t}} n_{\mathrm{p}}+\nabla \cdot\left(n_{\mathrm{p}} \mu_{\mathrm{p}} \mathbf{E}-D_{\mathrm{p}} \nabla n_{\mathrm{p}}\right)=S_{\mathrm{e}}, \\
& -\nabla^{2} \phi=\frac{e}{\epsilon_{0}}\left(n_{\mathrm{p}}-n_{\mathrm{e}}\right), \\
& \mathbf{E}=-\nabla \phi .
\end{aligned}
$$

In the above equations, $n_{\mathrm{e}}$ and $n_{\mathrm{p}}$ denote the number density of electrons and positive ions, respectively, where we assume 
only one ion type $\mathrm{N}_{2}{ }^{+}$to be formed. $\mathbf{E}$ represents the electric field, $\phi$ represents the electric potential and $e$ is the elementary charge. The mobilities (and diffusion coefficients) of electrons and positive ions are denoted by $\mu_{\mathrm{e}}$ (and $D_{\mathrm{e}}$ ) and $\mu_{\mathrm{p}}$ (and $D_{\mathrm{p}}$ ), respectively; they are assumed to be functions of the reduced electric field $E / N$, where $N$ is the gas number density. $S_{\mathrm{e}}$ is the impact ionization term in local field approximation.

The transport and reaction coefficients were taken from the work of Montijn et al. ${ }^{16)}$ and scaled to 80 bar.

At the cathode we implement secondary emission of electrons on ion impact, i.e.,

$$
n_{\mathrm{e}}=\gamma n_{\mathrm{p}},
$$

where $\gamma$ is the secondary emission coefficient whose value is taken to be 0.07 for simplicity.

\subsection{Model for the background gas}

To describe the gas dynamics, we adopt the compressible Euler equations ${ }^{17)}$ without viscosity, as in the studies by Tholin et al. ${ }^{11,18)}$ The source term for energy transfer from the electric discharge to the gas is Joule heating. ${ }^{11,19-21)}$ The system is closed with the ideal gas law. The relevant equations in cylindrical coordinates are

$$
\begin{aligned}
& \frac{\partial \rho}{\partial t}+\nabla \cdot(\rho \mathbf{v})=0, \\
& \frac{\partial\left(\rho v_{z}\right)}{\partial t}+\nabla \cdot\left(\rho v_{z} \mathbf{v}\right)=-\frac{\partial p}{\partial z}, \\
& \frac{\partial\left(\rho v_{r}\right)}{\partial t}+\nabla \cdot\left(\rho v_{r} \mathbf{v}\right)=-\frac{\partial p}{\partial r}, \\
& \frac{\partial \xi}{\partial t}+\nabla \cdot(\mathbf{v}(\xi+p))=\eta e\left(\mathbf{j}_{\mathrm{c}} \cdot \mathbf{E}\right),
\end{aligned}
$$

where $\mathbf{j}_{\mathrm{c}}$ is the conductive current density given by

$$
\mathbf{j}_{\mathrm{c}}=\mathbf{j}_{\mathrm{e}}+\mathbf{j}_{\mathrm{p}}
$$

where

$$
\begin{aligned}
& \mathbf{j}_{\mathrm{e}}=\mu_{\mathrm{e}} n_{\mathrm{e}} \mathbf{E}+D_{\mathrm{e}} \nabla n_{\mathrm{e}}, \\
& \mathbf{j}_{\mathrm{p}}=\mu_{\mathrm{p}} n_{\mathrm{p}} \mathbf{E}-D_{\mathrm{p}} \nabla n_{\mathrm{p}} .
\end{aligned}
$$

Here $\rho$ is the gas mass density, $\mathbf{v}$ is the gas velocity, $\xi=$ $\rho \epsilon+\frac{1}{2} \rho v^{2}$ is the total energy per unit volume, with $\epsilon$ being the internal energy per unit mass for the gas, $p$ is pressure, and $\eta$ is the fraction of discharge energy that contributes to gas heating. We have adopted the value of 0.3 for $\eta$ as suggested by Tholin et al. ${ }^{11)}$ based on physical arguments and fitting with experiments.

\subsection{Coupling between discharge and gas dynamics}

The electric discharge generates the Joule heat $\eta e\left(\mathbf{j}_{\mathrm{c}} \cdot \mathbf{E}\right)$ in the model for the gas. When the temperature rises, the gas expands, the gas number density $N$ decreases and hence the transport coefficients of the charged species and the reduced electric fields $E / N$ increase. There is a feedback in both directions (from discharge to gas and back) at every time step of the evolution.

\section{Simulation conditions}

In this work we adopt 3D-cylindrical geometry with azimuthal symmetry around the discharge axis $(r=0)$. The electrode configuration is plane-to-plane. The background

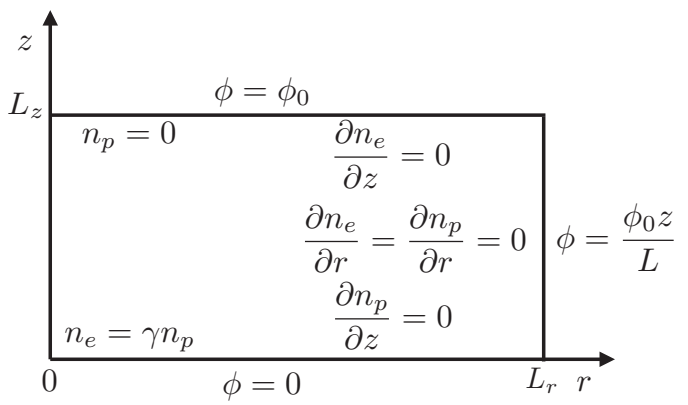

Fig. 1. Simulation setup: In the above figure, the gas medium is supercritical $\mathrm{N}_{2}$ at temperature of $300 \mathrm{~K}$ and pressure of 80 bar. The boundary conditions for charge densities (electronic/ionic) and for the electric potential are indicated. The gap length, $L$, is $0.3 \mathrm{~mm}$ and a dc-voltage, $\phi_{0}$ of $54 \mathrm{kV}$ is applied.

gas is taken to be supercritical $\mathrm{N}_{2}$ at a temperature of $300 \mathrm{~K}$ and a pressure of 80 bar. $^{7)}$ The applied electric field $\mathbf{E}=$ $-E \hat{\mathbf{e}}_{z}$ (where $E$ is the magnitude of the electric field and $\hat{\mathbf{e}}_{z}$ is the unit vector in the $z$-direction) drives the system. The electrons therefore move in the positive $z$-direction and the positive ions in the negative $z$-direction under the action of the field. The system length is $L=0.3 \mathrm{~mm}$ in $z$-direction and $3 L$ in $r$-direction.

\subsection{Boundary conditions}

We have cylindrical symmetry around the discharge axis $(r=0)$.

We set a homogeneous Neumann boundary condition for electron/ion densities onto the boundary in the radial direction. On the top boundary, the anode, we set positive ion density equal to zero, as ions are only produced within the gap and drift downward. Also, we set the perpendicular component of diffusive flux of electrons on the top boundary to zero.

Figure 1 shows the implemented boundary conditions. At the bottom electrode, the cathode, the perpendicular component of the ion-diffusive flux is set to zero. Also, since we include secondary electrode emission, the Dirichlet boundary condition (2) is imposed on the electron density at the cathode.

The electric potential is fixed as $\phi=0$ or $54 \mathrm{kV}$ on the lower $(z=0)$ or upper $(z=L)$ electrode. The electric potential varies linearly with $z$ on the right lateral boundary. In mathematical terms, the b.c. are

$$
\begin{aligned}
& \frac{\partial n_{\mathrm{e}}}{\partial z}\left(r, L_{z}, t\right)=0, n_{\mathrm{e}}(r, 0, t)=\gamma n_{\mathrm{p}}(r, 0, t), \\
& \frac{\partial n_{\mathrm{e}}}{\partial r}\left(L_{r}, z, t\right)=0, \\
& n_{\mathrm{p}}\left(r, L_{z}, t\right)=0, \frac{\partial n_{\mathrm{p}}}{\partial z}(r, 0, t)=0, \frac{\partial n_{\mathrm{p}}}{\partial r}\left(L_{r}, z, t\right)=0, \\
& \phi(r, 0, t)=0, \phi\left(r, L_{z}, t\right)=\phi_{0}, \phi\left(L_{r}, z, t\right)=\frac{\phi_{0} z}{L_{z}},
\end{aligned}
$$

where $L_{r}=3 L, L_{z}=L$, and $\phi_{0}=54 \mathrm{kV}$.

For the Euler equations, extrapolated boundary conditions ${ }^{22,23)}$ were assumed on all boundaries.

\subsection{Initial conditions}

We start the simulation with a Gaussian distribution of ions and electrons 

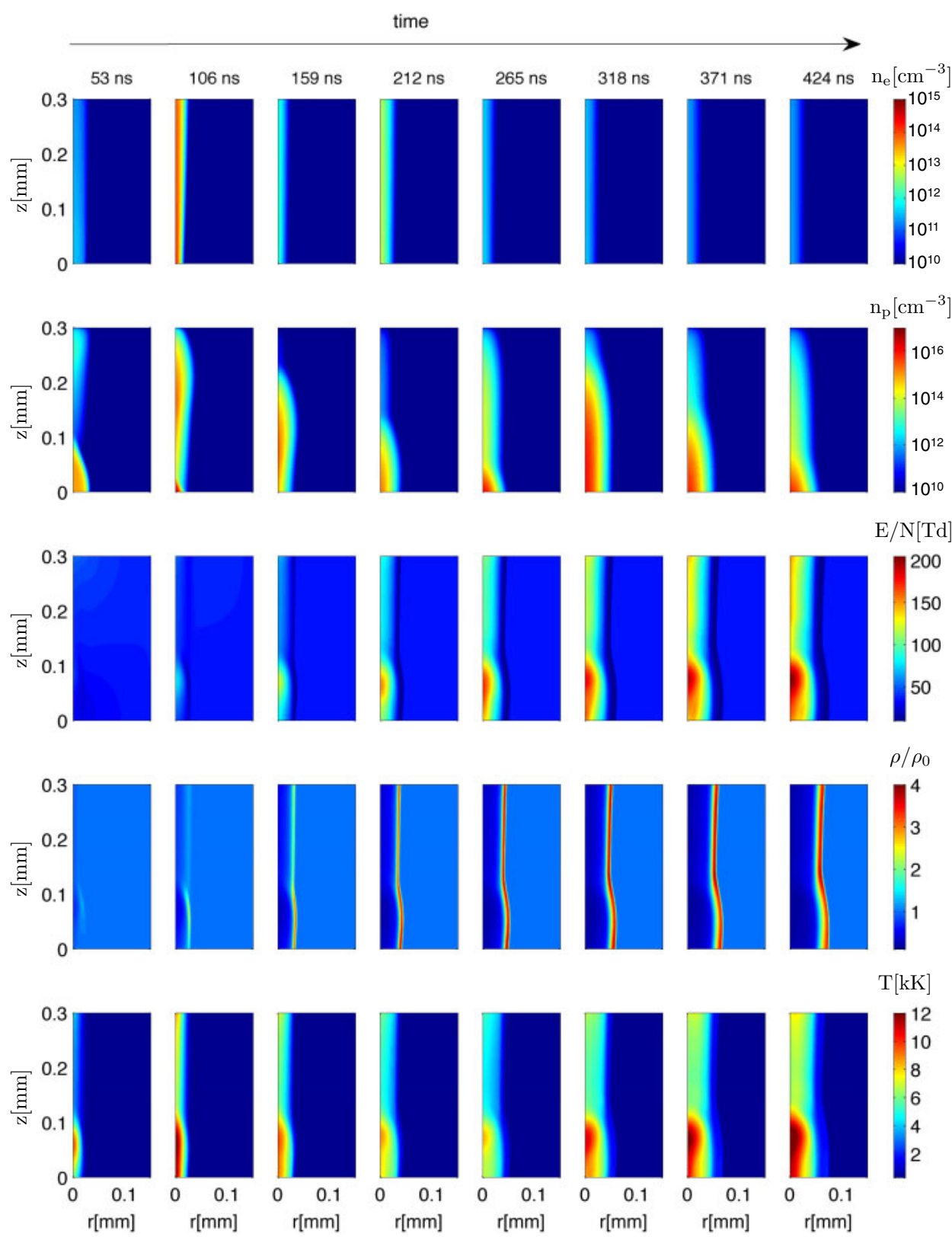

Fig. 2. (Color online) Evolution of electron number density $n_{\mathrm{e}}$, ion number density $n_{\mathrm{p}}$, reduced electric field $E / N$, gas density $\rho / \rho_{0}$ normalized to the density $\rho_{0}$ at $T=300 \mathrm{~K}$ and $80 \mathrm{bar}$, and gas temperature $T$. The initial seed of electrons and positive ions (16) is placed on the discharge axis $(r=0)$ at $z=0.09 \mathrm{~mm}$. The gas is nitrogen at $80 \mathrm{bar}$, and $54 \mathrm{kV}$ are applied to a gap of $0.3 \mathrm{~mm}$.

$$
\left.n(r, z)\right|_{t=0}=n_{0} \exp \left[-\frac{r^{2}}{\sigma_{r}^{2}}-\frac{\left(z-z_{0}\right)^{2}}{\sigma_{z}^{2}}\right],
$$

where we choose $n_{0}=5 \times 10^{11} \mathrm{~cm}^{-3}, z_{0}=0.09 \mathrm{~mm}, \sigma_{r}=$ $6.9 \mu \mathrm{m}$, and $\sigma_{z}=27.6 \mu \mathrm{m}$. This seed amounts to about 3700 electrons and ions. The initial gas temperature and pressure are chosen to be $300 \mathrm{~K}$ and $80 \mathrm{bar}$, respectively. Also, the gas velocity is chosen to be zero at the begining of the simulation.

\subsection{Numerical method}

The continuity equations of the discharge model are discretized with the MUSCL scheme ${ }^{24,25)}$ using the Koren limiter function. ${ }^{26)}$ Since we expect large gas density gradients in the system, we implemented a high-resolution scheme which although being computationally expensive is good at handling large gradients. For time-integration, explicit second-order
Runge-Kutta (midpoint rule) ${ }^{27)}$ is used. To solve Poisson's equation we used the FISHPACK solver. ${ }^{28)}$ Also, the Euler equations are discretized with the same numerical scheme as the discharge equations. A grid of 500 cells was taken along the $z$-axis and of $3 \times 500$ in the $r$-direction.

\section{Results and discussion}

We simulated the evolution of a discharge in nitrogen at 80 bar in a $0.3 \mathrm{~mm}$ gap with $54 \mathrm{kV}$ applied, and with the initial seed (9) of about 3700 electrons and ions. This seed might have been created by cosmic radiation or radioactivity, ${ }^{29)}$ but the possible formation process is beyond the scope of the current simulations.

Figure 2 shows the evolution of electron density $n_{\mathrm{e}}$, ion density $n_{\mathrm{p}}$, reduced electric field $E / N$, ratio of gas density over initial gas density $\rho / \rho_{0}$ and gas temperature $T$. We now discuss the rows of the figures sequentially. 
$n_{\mathrm{e}}$ : The electrons move much faster than the ions, and they are created by secondary emission at the cathode below and by impact ionization when they move upwards; therefore the electron density near the cathode is proportional to the local ion density and the growth of electron density in the $z$ direction is determined by the impact ionization rate.

$n_{\mathrm{p}}$ : An ion is generated together with every electron, but the ions move down more slowly. The time for the ions to cross the gap in the initial gas density and electric field is $172 \mathrm{~ns}$. When an ion wave reaches the cathode, new electrons are emitted and new ions and electrons are created in the gap. This refreshes the ion density and starts a new ion wave propagating downward.

$E / N$ : An important observation is that the electric field never changes much, i.e., the charge densities of electrons and ions are not sufficient to seriously modify the background electric field. The changes in the reduced electric field $E / N$ are due to the change of the gas density. In the last time step plotted at $t=424 \mathrm{~ns}$, the reduced electric field reaches a maximum of about $200 \mathrm{Td}$, while the breakdown reduced electric field in air is $120 \mathrm{Td}^{30)}$ We expect that the electric breakdown will continue after such high reduced fields have been reached.

In air, the breakdown field is defined as the value of the electric field at which the generation rate of electrons equals the attachment rate. In our case we have considered pure nitrogen in which attachment is absent. As air consists of $80 \%$ nitrogen, in practice the breakdown field in air provides a reasonable scale.

$\rho / \rho_{0}$ and $T$ : The temperature increases from 300 up to $12000 \mathrm{~K}$ mainly in the lower part of the system. Since the initial seed was located in the lower half, after the first time step the ions have drifted a little from their position but the electron density in the channel has been calculated all along the channel from Eq. (1a), starting from cathode. Electron density is essentially very small since the ions have not bombarded the cathode yet. Hence we see a concentration of heating at the position of the ions. This high temperature increases the pressure and creates an expansion shock front that can be seen in the density plot. At $t=159 \mathrm{~ns}$, the gas temperature has dropped on the lower part of the axis. In other words, the gas has cooled down. This happens due to the radial movement of shock wave.

\section{Summary and outlook}

We have simulated the evolution of a coupled system on electric discharge and background gas in a short plane-toplane electrode configuration in 2D-cylindrical symmetry. We have shown that with a simple model consisting of electron impact ionization and secondary electrode emission, simple relationships between the transport coefficients and gas density and heat transfer from the electric discharge to the gas, one can understand the formation of a spark. More specifically one can see how heat induced changes in the background gas density can trigger ionization in the discharge channel leading to breakdown. We see a pulsating behavior in the ion number density: the ions reach the cathode, liberate electrons from it and hence generate more electrons and ions in the gap. These ions propagate down to the cathode. This pulsating cycle continues until either the temperature induced density changes drive the reduced electric field to values above breakdown, or until the discharge activity stops.

We emphasize that this slow heat driven breakdown mechanism of short gaps is basically different from the fast streamer breakdown mechanism that is driven by space charge effects at the streamer head.

The current model was developed with the motivation to understand electrical breakdown with a simple physical model. Of course, one can broaden the model by further improvements, particularly by incorporating the complex relationship between transport coefficients and the gas density, and by further investigating the effects of temperature on the electron-ion source terms. These improvements will be part of future work.

\section{Acknowledgement}

A.A. acknowledges financial support under the "Computational sciences for energy research (CSER)" initiative by Shell and FOM (Dutch Physics funding agency) under project number 12CSER058.

1) K. Mochizuki, T. Ueno, T. Mizoguchi, S. Yanabu, S. Yuasa, and S. Okabe, Gaseous Dielectrics X (Springer, New York, 2004) p. 265.

2) N. H. Malik, A. A. Al-Arainy, and M. I. Qureshi, Electrical Insulation in Power Systems (Marcel Dekker, New York, 1997) p. 83.

3) S. A. Boggs, IEEE Electr. Insul. Mag. 5, 18 (1989).

4) A. Lee and L. S. Frost, IEEE Trans. Plasma Sci. 8, 362 (1980).

5) M. Hikita, S. Ohtsuka, N. Yokoyama, S. Okabe, and S. Kaneko, IEEE Trans. Dielectr. Electr. Insul. 15, 243 (2008).

6) J. Zhang, B. van Heesch, F. Beckers, T. Huiskamp, and G. Pemen, IEEE Trans. Plasma Sci. 42, 376 (2014).

7) J. Zhang, A. H. Markosyan, M. Seeger, E. M. van Veldhuizen, E. J. M. van Heesch, and U. Ebert, Plasma Sources Sci. Technol. 24, 025008 (2015).

8) N. A. Popov, Plasma Phys. Rep. 29, 695 (2003).

9) U. Ebert, S. Nijdam, C. Li, A. Luque, T. Briels, and E. van Veldhuizen, J. Geophys. Res. 115, A00E43 (2010).

10) A. Sun, J. Teunissen, and U. Ebert, J. Phys. D 47, 445205 (2014).

11) F. Tholin and A. Bourdon, J. Phys. D 46, 365205 (2013).

12) A. Komuro and R. Ono, J. Phys. D 47, 155202 (2014).

13) A. A. Kulikovsky, J. Phys. D 30, 441 (1997).

14) I. R. Rafatov, D. D. Sijacic, and U. Ebert, Phys. Rev. E 76, 036206 (2007)

15) D. D. Šijačić, U. Ebert, and I. Rafatov, Phys. Rev. E 71, 066402 (2005).

16) C. Montijn and U. Ebert, J. Phys. D 39, 2979 (2006).

17) E. F. Toro, Riemann Solvers and Numerical Methods for Fluid Dynamics: A Practical Introduction (Springer, Berlin, 2009) 3rd ed., p. 103.

18) F. Tholin and A. Bourdon, Plasma Sources Sci. Technol. 22, 045014 (2013).

19) O. Eichwald, M. Yousfi, P. Bayle, and M. Jugroot, J. Appl. Phys. 84, 4704 (1998).

20) O. Eichwald, M. Yousfi, P. Bayle, and M. Jugroot, J. Appl. Phys. 84, 4716 (1998).

21) J. A. Riousset, V. P. Pasko, and A. Bourdon, J. Geophys. Res. 115, A12321 (2010).

22) R. J. LeVeque, Finite Volume Methods for Hyperbolic Problems (Cambridge University Press, Cambridge, U.K., 2002) p. 129.

23) Web [http://www.clawpack.org/doc/bc.html].

24) A. Kurganov and E. Tadmor, J. Comput. Phys. 160, 241 (2000).

25) H. Nessyahu and E. Tadmor, J. Comput. Phys. 87, 408 (1990).

26) C. Montijn, W. Hundsdorfer, and U. Ebert, J. Comput. Phys. 219, 801 (2006).

27) W. Hundsdorfer and J. G. Verwer, Numerical Solution of Time-Dependent Advection-Diffusion-Reaction Equations (Springer, Berlin, 2003) p. 142.

28) Web [http://www.netlib.org/fishpack/].

29) S. Pancheshnyi, Plasma Sources Sci. Technol. 14, 645 (2005).

30) J. J. Lowke, J. Phys. D 25, 202 (1992). 